\title{
A CONTRIBUIÇÃO DA ANÁLISE EXPERIMENTAL DO COMPORTAMENTO PARA A FORMAÇÃO DO PROFISSIONAL EM PSICOLOGIA ENSINO DO BEHAVIORISMO ${ }^{1}$
}

\section{CONTRIBUTION OF EXPERIMENTAL ANALYSIS OF BEHAVIOR TO FORMATION OF PROFESSIONAL IN PSYCHOLOGY}

\author{
José Gonçalves MEDEIROS ${ }^{2}$ \\ Lígia Maria de Castro Marcondes MACHADO ${ }^{3}$ \\ Lucila CROSZEWICZ ${ }^{4}$ \\ Marina Bandeira de Pereira ACOST ${ }^{5}$
}

\begin{abstract}
RESUMO
O artigo relata as conclusões do Primeiro Encontro de Professores de Análise Experimental do Comportamento (AEC), realizado na Fundação de Ensino Superior de São João Dei Rey, em 1992, onde foram discutidos, entre outros, os seguintes temas: a) a contribuição da atividade de laboratório com animais para o ensino da Análise Experimental do Comportamento, no lugar de discutir como a atividade vem sendo realizada; b) o ensino da terapia comportamental; e c) o ensino dos processos básicos e as regras do método cientíifico.
\end{abstract}

\begin{abstract}
This is a report on the conclusions of the First Meeting of Professors of Experimental Analysis of Behavior (AEC), held in Fundação de Ensino Superior de São João Dei Rey, in 1992. The topics discussed were: a) what could or should be Ihe contribution af Ihe laboratory activities with anima is to the teaching of Ihe Experimental Analysis of Behavior rather than how the activity has been used; b) Ihe teaching of behavioral therapy; and c) Ihe teaching of the basic processes and methodology.
\end{abstract}

\footnotetext{
(1) Primeiro encontro de professores de Análise Experimental do Comportamento, realizado na Fundação de Ensino Superior de São João Dei Rey e organizado pelo Departamento das Psicologias daquela Instituição de Ensino Superior.

(2) Docente do Curso de Psicologia da UFSC (SC) e pesquisador do CNPq.

Endereço para correspondência: Caixa Postal 5060, CEP:88040-197, Campus Universitário, Florianópolis, SC. Email: Erro! Indicador não definido.

(3) Docente do Instituto de Psicologia da USP, relatora do GRUPO I, recentemente falecida.

(4) Docente do Curso de Psicologia da UFSC (SC), relatora do GRUPO II. E-mail: lucila@cfh.ufsc.br

(5) Docente do Curso de Psicologia da Fundação de Ensino Superiorde São João Dei Rey, MG, relatora do GRUPO III.
} 
O objetivo deste artigo é o de divulgar e discutir as conclusões do Primeiro Encontro de Professores de Análise Experimental do Comportamento. Reuniões anteriores já ocorreram com propósito semelhante. Uma delas ocorreu em 1979, por ocasião da realização da SBPC, em Fortaleza. Outra ocorreu no mesmo ano, na IX Reunião Anual de Psicologia da Sociedade de Psicologia de Ribeirão Preto $^{6}$ e da qual participaram Maria Amélia Matos, João Cláudio Todorov, Sérgio Luna e Rodolfo Azzi.

Luna (1979), em sua intervenção durante - Simpósio realizado em Fortaleza, na SBPC, aponta dois fenômenos: o primeiro diz respeito ao aumento do número de Faculdades com cursos de psicologia, com o consequente aumento do número de profissionais que se identificam com essa metodologia de trabalho, e o segundo diz respeito ao aumento de críticas que os trabalhos em AEC e seus autores vem recebendo, quer por parte dos alunos, quer por parte de outros profissionais. Para Luna nem sempre fazemos o que pregamos e nem sempre analisamos completamente antes de fazer. É muito comum, por exemplo, professores pregarem a necessidade de se analisar comportamentos dentro da tríplice contingência, porém não é muito fácil encontrar um professor capaz de descrever antecedentes e conseqüentes funcionais ou de utilizá-los para compreender o comportamento do aluno.

Os problemas apontados por Luna são, de fato, importantes. Contudo, eles não são exclusivos daqueles profissionais que lidam com 0 ensino da Análise Experimental. Haja vista os movimentos que ocorreram e que ocorrem em diversas Faculdades para a mudança dos currículos e do modo como são operacionalizados.

Como pode ser visto, o problema não é novo. Luna em sua intervenção discute o ensino da Análise Experimental nos últimos 15 anos (1964-1979). E desde a ocorrênciado Simpósio realizado em Fortaleza, até hoje, já se foram 20 anos (1979-1998).

\section{O Encontro de São João Del Rey}

Para este Primeiro Encontro foram convidados professores ${ }^{7}$ de diferentes Universidades do país que lidavam com o ensino de conteúdos referentes à disciplinas de cunho experimental (Psicologia Experimental, Modificação de Comportamento, Análise Experimental, etc.). Os participantes, de acordo com o tipo de atividade que desenvolviam em sua Universidade, organizaram-se em grupos e cada grupo discutiu um tema. Terminada a discussão, cada relator elaborou, a partir dos pontos discutidos, um relatório por grupo, cujo conteúdo segue transcrito neste artigo.

O Grupo I discutiu a "contribuição da atividade de laboratório com animais para o ensino da Análise Experimental do Comportamento, no lugar de discutir como a atividade vem sendo utilizada". Para isso, foi formulada a seguinte questão: QUEM É O PSICÓLOGO QUE QUEREMOS FORMAR ? Para a formação desse psicólogo: a) Qual é a contribuição da psicologia experimental? b) Qual é a contribuição de análise experimental do comporta-

\footnotetext{
(6) O texto completo desta mesa redonda está publicado nos Anais da IX Reunião Anual de Psicologia,1979,p.22-47.

(7) Participantes ligados à instituições de ensino: Universidade de São Paulo (USP): Lígia Maria Marcondes Machado e Carolina Martuscelli Bori; Fundação de Ensino Superior de São João Dei Rey (FUNREI): José Raimundo Facion, Marina Bandeira de Pereira Acosta e Roosevelt Riston Starling; Universidade Federal de Santa Catarina (UFSC): José Gonçalves Medeiros e Lucila Groszewicz; Universidade Federal de Minas Gerais (UFMG): Adélia Maria Santos Teixeira, Sílvia Rejane Castanheira Pereira e Sônia dos Santos Castanheira; Pontifícia Universidade Católica do Rio de Janeiro (PUC-Rio): Bernard Rangé; Universidade Federal do Pará(UFPa): Isabel Lúcia Florentino Conte; Centro de Ensino Superior de Juiz de Fora: Ivan Fajardo; Universidade Católica de Pelotas: Liliane Col; Universidade Federal de Juiz de Fora: Marcos Emanoel Pereira; Pontifícia Universidade Católica de Minas Gerais (PUC-MG): Sandra Maria de Castro Bernardes.

Participantes não ligados a instituições de ensino: Joinville (SC): Guilherme Guimbala Júnior; Divinópolis (MG): Jadir Raimundo da Silva; Campinas (SP): Magali Rodrigues Serrano; Belo Horizonte (MG): Sérgio Dias Cirino, Sônia Maria Guedes Gondim e Sérgio Dias Cirine.
} 
mento ? c) Qual é a contribuição da atividade de laboratório ? d) Qual é a contribuição da atividade de laboratório quando se utilizam animais como sujeitos?

O Grupo II discutiu: a) o "ensino da terapia comportamental e sua vinculação com a Análise Experimental do Comportamento;b) o hiato existente entre a prática da Análise Experimental do Comportamento e a prática da Terapia Comportamental. Neste sentido, algumas hipóteses foram levantadas: a) O ensino da Análise Experimental do Comportamento e da Análise Aplicada do Comportamentonão está habilitando suficientemente o aluno para uma formação em Terapia Comportamental; b) A prática da Terapia Comportamental necessita, muitas vezes, avançar além daquilo que é sustentado teoricamente; c) A Terapia Comportamental tem limites, precisando identificar e reconhecer estes limites; d) Pela forma como os currículos estão estruturados tem sido impossível formar terapeutas comportamentais dentro do curso, havendo, para isso, necessidade de organizar cursos de formação extra curriculares.

O Grupo III discutiu o ensino dos processos básicos e as regras dó método científico, apontando as seguintes questões: a) Dificuldades do aluno identificar no cotidiano (generalização) a ocorrência os princípios básicos que aprendeu nos textos clássicos; b) A influência negativa e preconceituosa de alguns professores de disciplinas ministradas anteriormente que, por desconhecimento ou má-fé, acabam por criar uma indisposição nos alunos contra a Analise Experimental do Comportamento; c) A ausência de disciplinas de cunho experimental nos períodos letivos seguintes ao ensino da Análise Experimental do Comportamento, que dêem seqüência ou continuidade à aprendizagem dos princípios básicos do comportamento em áreas de aplicação, seja ela clínica, escolar ou de pesquisa aplicada; d) Os conteúdos das disciplinas estão desatualizados, não levando em consideração as novas áreas de pesquisa, nem os novos campos de trabalho, impedindo o aluno de aplicar os conceitos aprendidos em áreas de educação, saúde,etc.;e) Dificuldades do aluno na assimilação de certos conceitos (p.ex. variável dependente e independente), decorrentes da falta de oportunidade de participação em projetos de pesquisa, onde ele tem oportunidade de identificá-los e de emprega-los corretamente; f) O laboratório não deve ser apenas uma ocasião de se fazer demonstrações de princípios básicos, nem ocasião apenas de praticar os exercícios básicos de laboratório, mas também uma passagem para o início do desenvolvimento de projetos de pesquisa e g) A Análise Experimental do Comportamento é ensinada isoladamente, não compartilhando com outras áreas do conhecimento para o desenvolvimento de investigações mais avançadas.

\section{GRUPO I : A UTILIZAÇÃO DE ATIVIDADES DE LABORATÓRIO COM ANIMAIS NO ENSINO DA ANÁLISE EXPERIMENTAL DO COMPORTAMENTO.}

Foi discutido neste Grupo a contribuição da atividade de laboratório com animais para o ensino da Análise Experimental do Comportamento, em lugar de discutir como a atividade vem sendo utilizada. Para fundamentar a discussão, foram formuladas algumas questões, com a proposta de respondê-las ao longo da reunião. As perguntas formuladas foram:

1) QUEMÉOPSICÓLOGOQUEQUEREMOS FORMAR?

Para a formação desse psicólogo:

a) Qual é a contribuição da psicologia experimental?

b) Qual é a contribuição de análise experimental do comportamento?

c) Qual é a contribuição da atividade de laboratório ?

d) Qual é a contribuição da atividade de laboratório quando se utilizam animais como sujeitos? 


\section{QUEM É O PSICÓLOGO QUE QUEREMOS FORMAR?}

Foi discutida a opção entre especialistas e generalistas. Pareceu claro a todos que, nos cursos em que lecionam, foi feita a escolha de se formarem psicólogos generalistas. Em parte, esta escolha refletiria uma ausência de definição e de compromisso quanto ao psicólogo que se deseja formar: a definição ficou por conta de cursos extra-curriculares e da atuação não sistemática em estágios.

Em função desta ausência de definição, procurou-se levantar algumas habilidades que seriam necessárias para a formação do psicólogo, qualquer que seja sua área de atuação e que, portanto, deveriam ser ensinadas em cursos que procuram formar generalistas. Assim, todo psicólogo deveria:

a) ficar sobcontrole do comportamento do organismo com o qual está trabalhando, seja o cliente para o psicólogo clínico, seja a escola para o psicólogo educacional etc.

b) ser capaz de analisar comportamento, descobrindo de que variáveis este é função;

c) ser curioso, no sentido de ser um colocador de perguntas;

d) ser crítico, especialmente perguntar-se de onde vem o conhecimento que constitui o corpo de dados da Psicologia.

Questão a): Qual é a contribuição da psicologia experimental ?

Tomando como referência este conjunto básico de habilidades, analisou-se como a Psicologia Experimental poderia contribuir para produzi-lo. Considerou-se que a Psicologia Experimental poderia tornar o estudante de Psicologia capaz de:

a) controlar e manipular variáveis,

b) ser controlado pelo dado produzido experimentalmente,

c) criticar os processos de produção do conhecimento, d) relativizar a importância das posições teóricas, considerando o caráter provisório do conhecimento.

Questão b): Qual é a contribuição de análise experimental do comportamento?

Considerou-se que o ensino da Análise Experimental do Comportamento poderia tornar o estudante capaz de:

a) organizar logicamente o dado produzido experimentalmente;

b) analisar comportamento, com base em pressupostos filosóficos e em um corpo de dados organizado;

c) utilizar um instrumental conceitual e uma maneira eficiente para trabalhar experimentalmente;

d) o fornecimento, pela Análise Experimental do Comportamento, de um modelo de desenvolvimento de um corpo teórico integrado e organizado, a partir da experimentação.

Questão c) Qual é a contribuição da atividade de laboratório ?

A atividade de laboratório foi analisada como tendo um papel importante, por permitir a ocorrência de comportamentos necessários para a formação do psicólogo com as características apontadas. Estes comportamentos seriam:

a) manipular e controlar variáveis;

b) fazer análise de comportamento;

c) colocar perguntas sobre as relações entre a variável independente (VI) e a variável dependente (VD);

d) colocar-se sob controle do comportamento do sujeito.

Questão d) Qualé a contribuição da atividade de laboratório quando se utilizam animais como sujeitos?

Foi considerado que o processo de instalação das habilidades básicas para a formação do psicólogo é um processo de modelagem, 
que poderia começar pela observação e manipulação do comportamento animal. Não só o animal utilizado, em geral o rato, é menos complexo que o homem como também o aluno lida com ele em situação padronizada e simplificada. Desta forma, é possível ter o aluno no início do curso de Psicologia, já executando controle e manipulação de variáveis, coleta e análise de dados no laboratório, utilizando animais como sujeitos.

\section{CONCLUSÃO}

Pareceu claro que o laboratório tem sido uma atividade usada principalmente para fornecer ao aluno uma oportunidade de "ver os princípios funcionando". Considerou-se que este uso constitui uma limitação desnecessária da potencialidade da atividade de laboratório. Julgou-se que ela poderia ser usada para treinar os comportamento que foram definidos como importantes, na direção de formar um psicólogo mais crítico e mais competente para analisar comportamento.

\section{GRUPO II: O ENSINO DA TERAPIA COMPORTAMENTAL.}

A discussão ocorrida no Grupo II pode ser resumida nos seguintes aspectos:

1. A Terapia Comportamental não pode desvincular-se da Análise Experimentaldo Comportamento, pois esta é sua base.

2. Parece haver um hiato entre a prática da Análise Experimental do Comportamentoe Terapia Comportamental e algumas hipóteses podem ser levantadas:

a) Oensino da Análise Experimental do Comportamento edaAnálise Aplicada do Comportamento não está habilitando o aluno para a formação em Terapia Comportamental;

b) Parece ser colocada muita ênfase na análise de dados em detrimento do ensino da habilidade para colocar em prática os princípios experimentais.

d) A Terapia Comportamentaltem limites e precisa identificar e reconhecer estes limites.

e) No momento, por questões talvez de tempo ou da maneira como estão estruturados os cursos de Psicologia, tem sido impossível formar terapeutas comportamentais durante ao graduação, havendo necessidade de organizar cursos extra faculdade para cobrir tal treinamento, o que parece ser o caminho, pois nem todos os alunos demonstram interesse ou aptidão para tal.

3. Fica em aberto para discussão posterior a seguinte questão: Quais as relações entre a Análise Experimental do Comportamentoe a Terapia Comportamentale, principalmente, com a pesquisa experimental.

Desta forma, a formação em Terapia Comportamentaladquire um desenvolvimento diferente da Análise Experimental do Comportamento. Incentivar o aluno para seguir cursos de pós-graduação seria um caminho, mas este é mais adequado para aformação do pesquisador acadêmico. Os cursos de Formação Terapêutica oferecem a oportunidade de formar 0 técnico, cujo papel tem seu lugar bem definido no mercado de trabalho. Na prática, as duas frentes de desenvolvimento ou os dois tipos de profissionais, são relevantes para a sociedade.

A questão principal que ficou como o ponto chave do Encontro foi: Qual a maneira de ensinar Análise Experimental do Comportamento, Análise Aplicada do Comportamento e Terapia Comportamental de modo a desenvolver as habilidades pertinentes nos nossos alunos, formando um corpo de conhecimentos coerente, sistematizado e bem fundamentado que possibilite uma prática eficiente? 


\section{GRUPO III: O ENSINO DOS PROCESSOS BÁSICOS E REGRAS DO MÉTODO CIENTÍFICO.}

As principais dificuldades do ensino da Análise Experimental do Comportamento levantadas pelos componentes deste grupo foram as seguintes:

Problema 1 : Dificuldade do aluno generalizar os princípios básicos que aprendeu nos textos clássicos (p. ex. Millenson, 1967) que se baseiam em estudos do comportamento animal para fazer um análise do comportamento humano no dia a dia. A passagem da compreensão do comportamento animal para o comportamento humano ocorre com dificuldades e os alunos, em geral, não vêem relação de um com o outro.

Foi levantada a sugestão de se adotar, desde o início, textos que utilizem exemplos do comportamento humano. $\mathrm{Na}$ Universidade $\mathrm{Fe}$ deral de Minas Gerais foi traduzido e está sendo utilizado, combons resultados, o texto de Baldwin e Baldwin (1986), "Princípios do comportamento na vida diária". Outra sugestão foi a de se montar um laboratório de estudo do comportamento humano.

Problema 2: Constatou-se a influência negativa de professores de disciplinas mais básicas, onde se estuda metodologia e as correntes teóricas da psicologia (ex. metodologia científica, história da psicologia, bases epistemológicas da psicologia), criando uma indisposição dos alunos contra a Análise Experimental do Comportamento. Como, em geral, os professores de AEC não estão presentes no ensino destas disciplinas, os alunos só recebem uma parte da informação, não tendo oportunidade de ouvir outras opiniões.

Foi levantada a necessidade de que os professores de Análise Experimental do Comportamento assumam uma parte destas disciplinas, proporcionando assim aos alunos um espaço no qual possam discutir e levantar questões sobre aspectos polêmicos que tenham sido mencionados por outros professores e/ou outras fontes de informação.

Um exemplo bem sucedido deste tipo de intervenção ocorre em Florianópolis, onde um professor de Análise Experimental do Comportamento leciona a parte referente ao Behaviorismo, em uma disciplina denominada Escolas I; em semestres subsequentes, em Escolas II e III se aborda respectivamente outras duas escolas diferentes, ou seja, Gestalt e Psicanálise. Parece, portanto, uma solução interessante que um representante de cada corrente se responsabilize pelo ensino de seu conteúdo.

Problema 3: Constatou-se a ausência de disciplinas avançadas, ou seja, nos períodos letivos seguintes ao ensino da Análise Experimental do Comportamento, que dêem uma seqüência ou continuidade à aprendizagem dos princípios básicos do comportamento em áreas de aplicação, seja ela clínica, escolar ou de pesquisa aplicada. Neste caso, o ensino da Análise Experimental do Comportamento fica sem utilidade pois não tem continuidade no curso de Psicologia.

Foi dado o exemplo do curso de Psicologia da Pontifícia Universidade Católica de Belo Horizonte onde há apenas uma disciplina básica de Análise Experimental do Comportamento, à qual não é dada continuidade com nenhuma outra posterior. Por outro lado, na UFMG há várias disciplinas iniciais dedicadas ao ensino de Análise Experimental do Comportamento, mas não tem nenhuma posterior que lhe dê continuidade. Na Fundação de Ensino Superior de São João Dei Rey há duas disciplinas de Análise Experimental do Comportamento, seguida posteriormente por apenas uma disciplina de Terapia Comportamental nos últimos períodos.

Foi sugerido que o ideal seria uma seqüência de disciplinas gradualmente mais avançadas, dando continuidade ao ensino básico, que culminasse em áreas de aplicação. Esta continuidade levaria para as diferentes áreas a 
maneira científica de trabalhar com dados que a Análise Experimental do Comportamento proporciona, culminando portanto com a pesquisa de aplicação.

Um modelo parcial deste tipo de continuidade seria o caso de Belém, que apresenta três níveis de disciplinas (básico, avançado I e avançado II), seguidos de dois níveis de estágio supervisionado de pesquisa (I e II) sem, no entanto, culminar em pesquisa de aplicação. Isto deixa, portanto, ainda em aberto o hiato entre o básico e a aplicação.

Problema 4: Os conteúdos dos cursos estão muito caducos. Estamos repetindo os mesmos conteúdos sem nenhuma renovação, sem atualização baseada nas novas áreas de pesquisa emergentes, nem em novos campos de trabalho. Isto impede ao aluno a possibilidade de aplicar os conceitos aprendidos em áreas de educação, saúde, etc.

Problema 5: A dificuldade do aluno em assimilar certos conceitos (ex. variável dependente e independente), foi vista como decorrente da falta de oportunidade do aluno de utilizalos em projetos de pesquisa, onde ele precisa identificar estes conceitos, praticando assim sua habilidade de empregá-los corretamente. Não se trata de conceitos que são aprendidos apenas lendo-se um texto.

Problema 6: O laboratório não deve ser apenas uma ocasião de se fazer demonstrações de princípios básicos, nem ocasião apenas de praticar os exercícios básicos de laboratório, mas também uma passagem para o início do desenvolvimento de projetos de pesquisa. $\mathrm{O}$ aluno deve ter, portanto, a oportunidade de participar de projetos de pesquisado professor, fazer auto-experimentação, fazer replicações diretas de pesquisas, etc...

Problema 7: O ensino da Análise Experimental do Comportamentoé dado isoladamente, não compartilhando com outras áreas do conhecimento para o desenvolvimento de investigações mais avançadas. Atualmente pesquisas de ponta estão sendo desenvolvidas em áreas de superposição entre dois ou mais campos do conhecimento.

Procurou-se identificar as áreas do conhecimento que poderiam contribuir para o ensino da Análise Experimental do Comportamento, tendo sido sugerido, por exemplo, o ensino da teoria da evolução.

\section{CONCLUSÃO}

Cinco anos se passaram após este Primeiro Encontro e, apesar do desejo da Professora Lígia, não houve oportunidade de novo encontro para continuidade da discussão e constatação das mudanças que eventualmente tenham ocorrido em nosso repertório acadêmico de ensinar. Temos a certeza de que a oportunidade que tivemos, embora única, tornou possível uma revisão no tipo de trabalho que vínhamos fazendo.

Há ainda a ressaltar a importante contribuição de Hayese Hayes (1992), especialmente com o livro Understanding verbal relations, que muito auxilia naquilo que considerávamos um vazio entre a Análise Experimental do Comportamento e a Terapia Comportamental. Estes avanços, bem como os recentes resultados decorrentes dos estudos de equivalência de estímulos, vem demonstrando que a Análise Experimental do Comportamento e a Análise Aplicada do Comportamento tem ainda muito a oferecer para a consolidação da Psicologia como uma ciência objetiva.

São exemplos de pessoas inovadoras e entusiastas como a Professora Lígia que necessitam ser imitados para que continuemos questionando, trocando e buscando novas maneiras de ensinar e pesquisar e aprimorando nossa ferramenta de trabalho para construir e consolidar a Análise Experimental do Comportamento como a Ciência do Comportamento Humano. 


\section{REFERÊNCIAS}

BALDWIN, J. D. e BALDWIN, J. I. (1986). Behaviorprinciples in everyday life. (trad., para efeito de circulação interna,como título de Princípios do comportamento na vida diária, pelos profs. da UFMG, LauraF. Ciruffo, Maria José E. Vasconcelios, Sílvia R.Castanheira Pereira e SôniaS. Castanheira).Santa Bárbara: Universidade da Califórnia.

HAYES, L. J. e HAYES, S.C. (1992). Understanding verbal relations: the Second and Third International Institute on Verbal Relations. Reno (Nevada): Context Press.

LUNA, S.V. (1979). Uma revisão crítica de quinze anos de ensino da análise experimental do comportamento no Brasil: propósitos, efeitos e implicações. Texto de circulação interna referente ao Simpósio realizado durante a 31 ReuniãoAnualdaSBPC, Fortaleza, Ceará.
MACHADO, L. M. M.; BANDEIRA, M. P. A. e CROSZEWICZ, L. (1992). Relatório consolidado do I Encontro de Professores de Análise Experimental do Comportamento, realizado em São João Del'Rey.

MATOS, M. A.; TODOROV, J. C.; LUNA, S. V. e AZZI, R. (1979) Análise Experimental do Comportamento: avaliação crítica. Anais da IX Reunião Anual de Psicologia da Sociedade de Psicologia de Ribeirão Preto, p.22-47.

MILLENSON, J. R. (1967) Princípios de análise do comportamento (trad. de Alina de Almeida Souza e Dionede Rezende). Brasília:Coordenada Editora de Brasília. 